\title{
Equivalent currents associated with morning-sector geomagnetic Pc5 pulsations during auroral substorms
}

\author{
K. Kauristie ${ }^{1}$, M. V. Uspensky ${ }^{1}$, N. G. Kleimenova ${ }^{2}$, O. V. Kozyreva ${ }^{2}$, M. M. J. L. Van De Kamp ${ }^{1}$, S. V. Dubyagin ${ }^{1}$, and \\ S. Massetti $^{3}$ \\ ${ }^{1}$ Finnish Meteorological Institute, Helsinki, Finland \\ ${ }^{2}$ Institute of Physics of the Earth, Russian Academy of Sciences, Moscow, Russia \\ ${ }^{3}$ Institute for Space Astrophysics and Planetology, National Institute of Astrophysics, Rome, Italy \\ Correspondence to: K. Kauristie (kirsti.kauristie@ fmi.fi)
}

Received: 11 September 2015 - Revised: 29 February 2016 - Accepted: 9 March 2016 - Published: 7 April 2016

\begin{abstract}
Space and time variations of equivalent currents during morning-sector Pc5 pulsations $(T \sim 2-8 \mathrm{~min}$ ) on 2 days (18 January and 19 February 2008) are studied in the context of substorm activity with THEMIS and MIRACLE ground-based instruments and THEMIS P3, P5, and P2 probes. These instruments covered the 22:00-07:00 magnetic local time during the analyzed events. In these cases abrupt changes in the Pc5 amplitudes, intensifications and/or weakenings, were recorded some minutes after auroral breakups in the midnight sector. We analyze three examples of Pc5 changes with the goal to resolve whether substorm activity can have an effect on Pc5 amplitude or not. In two cases (on 19 February) the most likely explanation for Pc5 amplitude changes comes from the solar wind (changes in the sign of interplanetary magnetic field $B_{z}$ ). In the third case (on 18 January) equivalent current patterns in the morning sector show an antisunward-propagating vortex which replaced the Pc5-related smaller vortices and consequently the pulsations weakened. We associate the large vortex with a field-aligned current system due to a sudden, although small, drop in solar wind pressure (from 1 to $0.2 \mathrm{nPa}$ ). However, the potential impact of midnight substorm activity cannot be totally excluded in this case, because enhanced fluxes of electrons with high enough energies $(\sim 280 \mathrm{keV})$ to reach the region of Pc5 within the observed delay were observed by THEMIS P2 at longitudes between the midnight and morning-sector instrumentation.
\end{abstract}

Keywords. Magnetospheric physics (current systems)

\section{Introduction}

During auroral substorms the strongest geomagnetic activity and auroras appear typically in the midnight sector of the auroral oval (McPherron, 1979; Akasofu, 1964). Some studies have shown, however, that sudden changes in the conditions in other sectors of the auroral oval may happen simultaneously with the substorm breakups at midnight. Statistical surveys have shown that the auroral electrojets in dawn and dusk sectors can intensify (Borälv et al., 2000), and morning- or noon-sector geomagnetic pulsations can either intensify or weaken (Samson and Rostoker, 1981; Rostoker et al., 1984) at substorm breakup times or soon after them. Whether there is a real cause-effect relationship between substorm breakups and activity changes in dawn and dusk sectors or whether both changes are just driven by the same alteration in solar wind is one of the open topics in solar-terrestrial physics.

Coordinated analysis of ground-based and satellite observations can be used to address the problematics of coincidental activity changes in midnight and other sectors of the oval. In the case of substorm-electrojet linkage Borälv et al. (2000) come to the conclusion that the cause-effect relation exists, and they attribute the transfer of information from midnight sector to dawn and dusk to rapid changes in the convection electric field. Connors et al. (2003) demonstrate how morning-sector Ps6 pulsations and the related omega bands can appear simultaneously with substorm onset at midnight. Ps6 is a fluctuation with a period of $10-40 \mathrm{~min}$. They are seen primarily in the east component of magnetic field in the post-midnight sector during the recovery phase of substorms 
and during steady magnetospheric convection (McPherron, 2005). Also in this case the electric field intensification at the time of onset is suggested to trigger the Ps6 activity in the morning sector. In the above-mentioned early studies on the substorm-pulsation linkage, Rostoker et al. (1984) suggest that interplanetary magnetic field (IMF) $B_{z}$ (north-south component) changes to positive values trigger both the substorm and the favorable conditions for morning-sector pulsations. The case study of storm time pulsations by Kleimenova et al. (2005) demonstrates how density variations in the solar wind can either enhance or suppress morning-sector pulsations synchronously with midnight substorms or with less irregular pulsation bursts in the evening sector.

The morning-sector geomagnetic pulsations discussed by Rostoker et al. (1984) and Kleimenova et al. (2005) are socalled Pc5 pulsations, whose typical periods are in the range of $\sim 2-8 \mathrm{~min}$. Pulsations have been studied extensively now for more than forty years (for reviews on previous studies, see Baker et al., 2003, and McPherron, 2005). They are caused by ultra-low frequency (ULF) plasma waves appearing in the magnetosphere and driven either by ULF waves in the solar wind (e.g., Kepko et al., 2002; Fenrich and Waters, 2008; Stephenson and Walker, 2010; Zelinsky et al., 2014) or by Kelvin-Helmholtz instability (KHI) building up at the magnetopause where velocity shear between magnetosheath and magnetospheric plasma velocities can appear (e.g., Mann et al., 1999, and references therein). The generation of Pc5 pulsations is often connected with excitation of resonance oscillations of magnetic field lines (field-line resonances, FLRs) in the magnetosphere (Samson et al., 1992; Ziesolleck and McDiarmid, 1995). FLRs can appear in two modes, toroidal and poloidal. In the toroidal mode, which is more common, field-line displacements take place in the longitudinal direction.

The extensive statistical study on Pc5 activity by Baker et al. (2003) reveals that FLRs appear preferentially in the morning sector at high latitudes and when the solar wind speed is high (more than $\sim 550 \mathrm{~km} \mathrm{~s}^{-1}$ ). Their occurrence probability is slightly higher for positive than for negative IMF $B_{z}$. In the traditional schematic description resonating field lines are compared to vibrating strings whose ends are attached to the high-latitude ionosphere at the Northern and Southern hemispheres. Standing plasma waves appearing, for example, in the cavity between magnetopause and plasmapause feed energy to the vibrating field lines. Vibrations are strongest at those field lines which have the correct length and plasma density for resonant interaction with the cavity waves. In a more realistic theoretical description the cavity is replaced with a waveguide which is open in the downtail direction. Furthermore, the outer boundary at magnetopause is modeled with a free boundary allowing energy exchange between magnetosphere and magnetosheath flows (Mann et al., 1999; Mann and Wright, 1999).

The magnetic field variations, Pc5 pulsations and substorm breakups, observed at the ground are associated with the current system which the magnetohydrodynamic (MHD) waves of FLRs generate (Scoffield et al., 2005). This system consists of a set of field-aligned currents (FACs) with varying directions according to latitude and the associated Hall and Pedersen currents in the ionosphere (cf. Fig. 19 in McPherron, 2005). The latitude of resonant field lines can be located with data from a meridional ground-based magnetometer chain: the amplitude of oscillations is largest and the phase has a change of $180^{\circ}$ at the FLR footpoint. In this region also the variations in the ionospheric electric field are strongest (Scoffield et al., 2005).

One attempt to address the issue of substorm-Pc5 connection is presented in the paper by Kauristie et al. (2013; hereafter KKetal2013) where two pulsation intervals, during 04:00-06:00 UT on 18 January and 19 February 2008, were analyzed with a collection of ground-based and satellite data. In the present study we continue the analysis of KKetal2013 by studying dynamics of morning-sector ionospheric currents during their Pc5 events. We present equivalent current distributions as derived from the Fennoscandian International Monitor of Auroral Geomagnetic Effects (IMAGE) magnetometer chain data (Viljanen and Häkkinen, 1997) with the method of spherical elementary current systems (SECSs, Amm and Viljanen, 1999). Our goal is to investigate whether there are some changes in the regional current patterns which would give new information about the differences between the Pc5 intensifications and fadings observed by KKetal2013.

In the next section we give a brief summary of the KKetal2013 study. After that we introduce the SECS method and present results from the IMAGE data analysis. A summary of our main findings and concluding remarks are presented in Sect. 5.

\section{The example events on 18 January and 19 February 2008}

In KKetal2013 two pulsation periods, during 04:0006:00 UT on 18 January and 19 February 2008, were analyzed with an extensive set of ground-based and satellite data from the NASA Time History of Events and Macroscale Interactions during Substorms (THEMIS) mission (Angelopoulos, 2009; Mann et al., 2008) observing the midnight sector and with Fennoscandian (Magnetometers - Ionospheric Radars - All-sky Cameras Large Experiment, MIRACLE) and Greenland ground-based instrumentation (Syrjäsuo et al., 1998; Friis-Christensen et al., 1985; Hagfors, 1982; Röttger, 1991) which was monitoring the morning-sector activity (cf. Fig. 1). The solar wind conditions as observed by the Geotail satellite during both events are discussed below in Sect. 4.3. The two events are interesting siblings in the sense that the constellation of spacedbased and ground-based instrumentation happened to be essentially the same for both cases which themselves resem- 


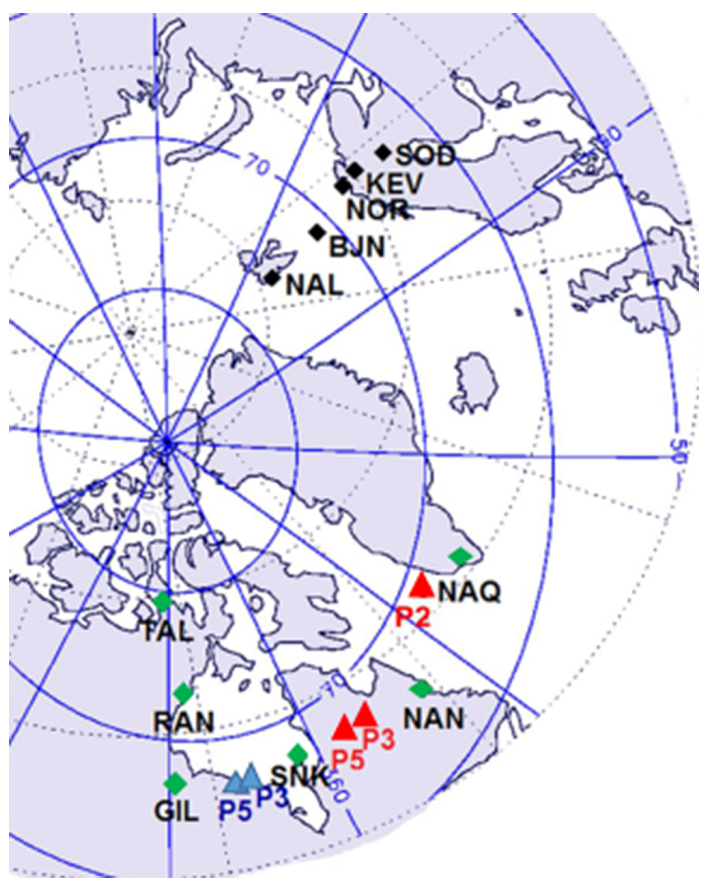

Figure 1. Instrumentation used to study the substorm events on 18 January and 19 February 2008. The magnetic conjugacy points of THEMIS probes $\mathrm{P} 5$ and $\mathrm{P} 3$ are shown with blue and red triangles (triangles in red correspond to conjugacy points of the 18 January event). During both events the locations of THEMIS instrumentation (TAL, RAN, GIL, P3, P5) were at 21:00-23:00 MLT and that of the Fennoscandian instrumentation (NAL, BJN, NOR, KEV, SOD) was 05:00-07:00 MLT (adapted from Kauristie et al., 2013).

bled each other in several respects. In both cases Pc5 activity showed typical FLR features as driven by high-speed solar wind and the properties of pulsations experienced rapid modifications within $2-4$ min delays, i.e., roughly within the pulsation period time, from substorm breakups which took place in the midnight sector.

Figures 2 and 3 summarize the mutual timing of substorm activity and Pc5 pulsations as reported in KKetal2013. The periods of Pc5 weakenings and intensifications are marked with blue and red boxes and shadings in the figures. During the first substorm (Fig. 2) a clear weakening of Pc5 was observed in the morning sector some minutes after an auroral breakup at $\sim 22: 00$ magnetic local time (MLT) and $\sim 67^{\circ}$ magnetic latitude (MLAT) (GIL, cf. Fig. 1, auroral data not shown here) at 05:11 UT. In THEMIS ground-based magnetometer data the substorm expansion phase activity is seen as negative deviation in magnetic north component starting at the same time as the auroral breakup at GIL but being recorded first at station SNK and only some minutes later at GIL. During the second substorm (Fig. 3) the first auroral breakup was observed, at GIL at 05:25 UT and a further intensification in auroras and ionospheric currents took place 12 min later at MLAT $\sim 74^{\circ}$ (RAN). The morning-sector pul-

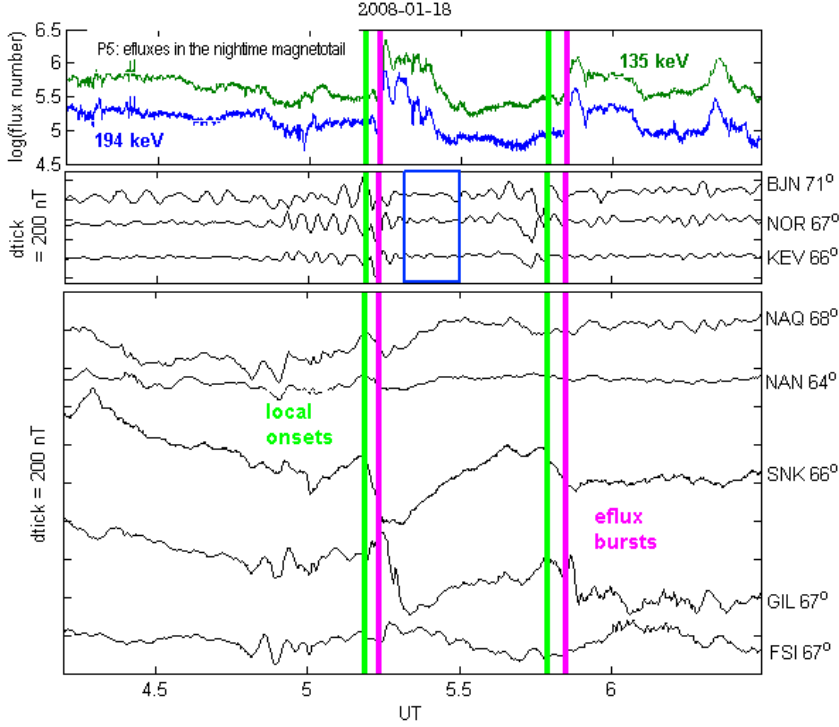

Figure 2. Ground-based and satellite observations during the substorm event on 18 January 2008. From top to bottom, electron fluxes as measured by the THEMIS Probe 5 (channels 135 and $194 \mathrm{keV}$ ), geomagnetic pulsations at Fennoscandian stations BJN, NOR, KEV, magnetometer data from Greenland (NAQ) and eastern Canada (NAN), and magnetometer data from the THEMIS groundbased stations. The time of Pc5 decrease is marked with a blue box (adapted from Kauristie et al., 2013).

sations intensified at the time of the first breakup, while the auroral intensification at RAN was followed by a decrease in Pc5, not as intensive as in the case of 18 January 2008, but a clear local weakening anyway.

Energetic electron flux measurements by THEMIS satellites (P3, P4, and P5) in the magnetotail at distances 9-11 Earth radii $\left(R_{\mathrm{e}}\right)$ showed increases some 2-4 min after the activations in all the three cases. This suggests that the initial onset regions were either in the center or on western side of the THEMIS satellites as electrons drift to the east from the longitude of substorm onset injection (Li et al., 1998, 2003). An alternative explanation for the flux enhancements can be leakages of electrons from the outer radiation belt due to changes in the magnetic topology (Sergeev et al., 2008) in which case estimating the onset longitude and timing is not straightforward. The THEMIS satellite $\mathrm{P} 2$, which was longitudinally separated with $\sim 50^{\circ}$ eastward (westward) from the other satellites on 18 January (19 February), showed electron (ion) flux enhancements with energy dispersion soon after the flux enhancements in the midnight sector. An interesting coincidence is that the electron flux increases at THEMIS satellites took place systematically around the same times as the Pc5 intensity changes took place in the morning sector. THEMIS P2 and P5 electron flux data are discussed in more detail below (in Sect. 4.3).

During both substorm events some signatures of eastwardpropagating auroras were observed in THEMIS all-sky cam- 


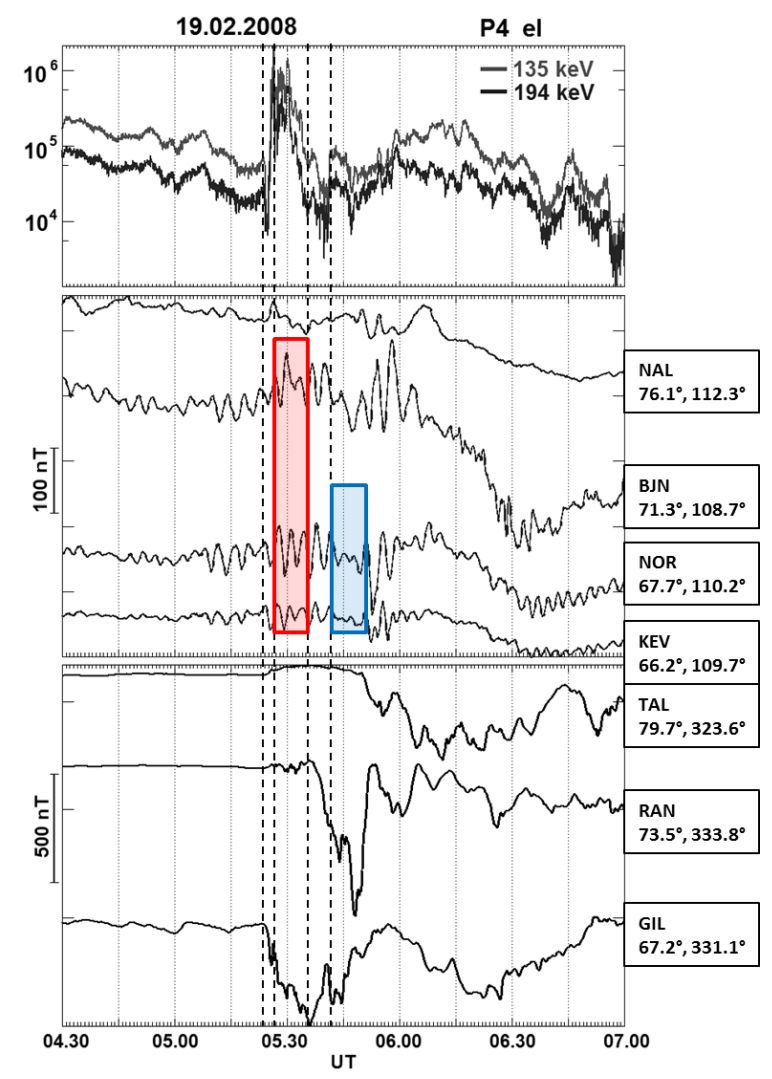

Figure 3. Ground-based and satellite observations during the substorm event on 19 February 2008. From top to bottom, electron fluxes as measured by the THEMIS Probe 5, geomagnetic pulsations at Fennoscandian stations BJN, NOR, KEV, magnetometer data from Greenland (NAQ) and eastern Canada (NAN), and magnetometer data from the THEMIS ground-based stations. The times of Pc5 intensifications and weakenings are marked with red and blue shadings (adapted from Kauristie et al., 2013).

eras (ASCs) during the cases of Pc5 fading while in the case of Pc5 intensification such auroras were missing. Although the eastward-propagating auroras were rather dim, they in some sense resemble so-called "auroral horns", which have been studied, for example, by Koskinen et al. (1990). Their study suggests that the ionospheric signature of a magnetosonic wave can be an auroral horn propagating either westward of eastward from the breakup region. With typical magnetosonic wave propagation speeds information can spread from the midnight sector to dawn sector in $\sim 4$ min (Borälv et al, 2000), which is a similar delay time as observed in the cases of 18 January and 19 February 2008. However, if such wave propagation really took place, for example, on 18 January, it did not cause any obvious signatures for Greenland ground-based magnetometers (cf. NAN and NAQ magnetograms in Fig. 2).

\section{Deriving ionospheric equivalent currents from ground-based magnetometer data}

Any vector field appearing on a spherical surface can be represented as a combination of two vector fields, one divergence-free and the other is curl-free. This is true also for the horizontal ionospheric currents which we here assume to flow in a thin sheet at $100 \mathrm{~km}$ altitude. From the current continuity equation it follows that FACs are linked with the curl-free part of horizontal currents and together these currents form a poloidal current system whose impact cannot be measured by ground-based magnetometers (Amm, 1997). On the contrary, the divergence-free part of horizontal currents $\left(J_{\mathrm{df}}\right)$, which is also called equivalent currents, can be measured with ground-based instrumentation. In the first approximation (with absence of strong conductance gradients) the curl-free and divergence-free parts of horizontal currents can be associated with Pedersen and Hall currents, respectively.

If the ratio of Hall to Pedersen conductance (often called $\alpha$ parameter) can be assumed to be a constant and the gradient of Hall conductance to be mainly in the direction of ionospheric electric field, then the FAC density can be estimated with the following formula (Juusola et al., 2009):

$\boldsymbol{j}_{\mathrm{FAC}}=-\frac{1}{\alpha}\left(\nabla \times J_{\mathrm{df}}\right)$.

In this study we will not use Eq. (1) for $\boldsymbol{j}_{\mathrm{FAC}}$ intensity estimates as we do not have any reasonable means to estimate $\alpha$, but we will keep in mind that clockwise (counterclockwise) vortices in equivalent current patterns (see below) can be associated with downward (upward) FAC locations which are roughly in the center of the vortices.

In the approach developed by Amm (1997) and Amm and Viljanen (1999) the two components of ionospheric currents are represented with two different types of SECSs. For mathematical and graphical representation of these systems, see, e.g., Juusola et al. (2006). For creating two-dimensional (2D) equivalent current patterns for the Fennoscandian region we use a grid of divergence-free elementary current systems, where the poles of the systems cover the latitude-longitude region from $53.9^{\circ} \mathrm{N}, 2.5^{\circ} \mathrm{E}$ (south-west edge) to $83.9^{\circ} \mathrm{N}$, $43.1^{\circ} \mathrm{E}$ (north-east edge). The distances of the poles in the grid are 0.6 and $1.4^{\circ}$ in latitudinal and longitudinal directions. The intensities of the elementary currents are determined so that they provide the minimum error match with the IMAGE magnetometer recordings. Equivalent currents are computed in a grid with latitudes from 59.0 to $79.4^{\circ} \mathrm{N}$ and longitudes from 3.2 to $42.4^{\circ} \mathrm{E}$. In this grid the latitudinal and longitudinal resolutions are the same as those in the grid of elementary current poles. In the studies on auroral electrojets we use latitude-vs.-time plots where the intensity and direction of the electrojet is defined by the east-west component of the equivalent currents along the central meridian $\left(22.1^{\circ} \mathrm{E}\right)$ of the 2-D equivalent current patterns. 
The approach which we use here for deriving equivalent currents is exactly the same as that used for generating the 10-year database of equivalent currents in the EU FP7 ECLAT (European Cluster Assimilation Technology) project (Juusola et al., 2014). ECLAT produced several data products from ground-based instrumentation to support analysis of Cluster satellite mission data. These data products are now available in the Cluster Active Archive (http://caa.estec.esa. int/caa/). When studying relatively weak equivalent currents, like is the case in our Pc5 event on 19 February 2008, it is important to determine the baseline levels for magnetometer data with special attention. For the baseline determination we have used the routines developed in ECLAT (van de Kamp, 2013), which do a careful job in eliminating the impact of quiet time current systems from magnetometer data and handle the north and east components of the magnetic field separately.

\section{Results from the SECS analysis}

Figures 4 and 5 present the electrojet variations in time and latitude during 04:00-06:00 UT on 18 January and 19 February 2008. Westward (eastward) equivalent currents are shown with blue (red) colors in the plots. The two substorm events took place in slightly different solar wind conditions. The solar wind speed (shown in Sect. 5.1) was higher on 18 January $\left(\sim 700 \mathrm{~km} \mathrm{~s}^{-1}\right)$ than on 19 February $\left(\sim 620 \mathrm{~km} \mathrm{~s}^{-1}\right)$ which most likely is the reason for the intensity of pulsations to be larger in the first event than in the second one (note the different scales in the color palettes of Figs. 4 and 5). During the first event IMF $B_{z}$ is mostly positive (cf. Fig. 9) and consequently the electrojet activity stays roughly at the same latitudes during the whole period. During the second event IMF $B_{z}$ was mainly negative during 04:30-05:10 UT (cf. Fig. 10), and thus dayside reconnection was ongoing and caused polar cap expansion, which is visible in Fig. 5 as a gradual shift of the electrojet to lower latitudes. As demonstrated, for example, by Hubert et al. (2010) the poleward boundary of morning-sector westward equivalent currents can sometimes be used as a proxy for the open-closed field-line boundary (polar cap boundary). Estimating the polar cap location and its motion is not straightforward in our first event (Fig. 4), because pulsation activity was dominating over the background electrojet activity. However, according to auroral camera data from Ny-Ålesund (NAL), the polar cap boundary was poleward of $79^{\circ} \mathrm{N}$ (geographical latitude), as auroras were observed in the northern sky of the station during the whole period.

The geomagnetic pulsations appear in Figs. 4 and 5 as sequences of blue and red temporal increases (blobs) in two latitudinal bands. Typically, when a blob in the band of lower latitude is red, then the simultaneous blob in the higherlatitude band is blue (and vice versa). Such structures correspond to vortices in 2-D equivalent current plots. When a

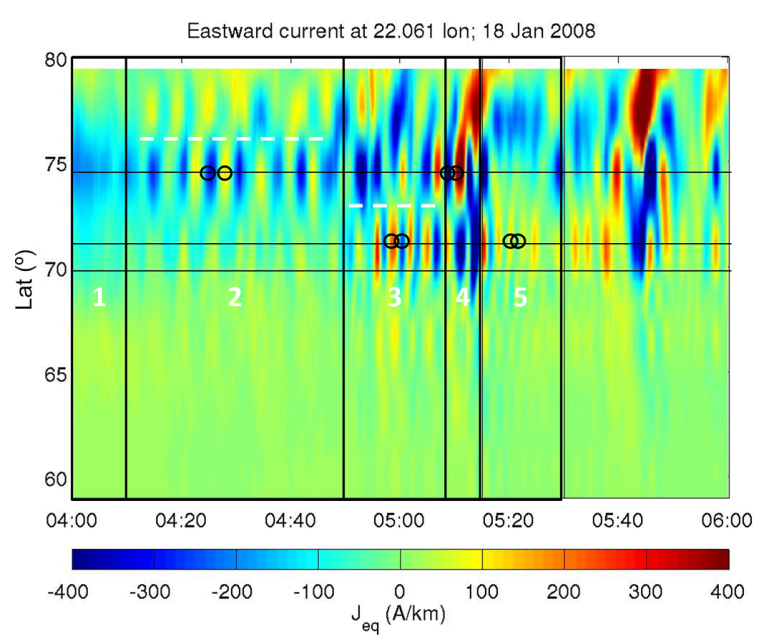

Figure 4. Eastward equivalent currents along the geographic meridian $22.1^{\circ}$ E during 04:00-06:00 UT on 18 January 2008. (The vertical axis is in geographical latitudes.) The five periods with different characteristics in 2-D equivalent current patterns are marked with black boundaries and white numbering. The geographic latitudes of stations BJN, NOR, and KEV are marked with the black horizontal lines from top to bottom, respectively. The auroral breakup at midnight sector took place at the beginning of Period 4. The white dashed lines show the maximum FLR latitude based on Fourier analysis of 3.1 and $5.2 \mathrm{mHz}$ magnetic variations. The time instants of the 2-D equivalent current maps of Fig. 6 are shown with the black circles.

blue (red) blob is at higher latitude than the red (blue) blob, then there is a counterclockwise (clockwise) vortex in the equivalent currents, when viewed from above. As counterclockwise (clockwise) vortices appear typically at the footpoints of upward (downward) directed FACs, Figs. 4 and 5 confirm the previously known fact that Pc5 and FLR activity is associated with oscillating FAC systems.

The geographic latitudes of IMAGE stations BJN $\left(74.5^{\circ} \mathrm{N}\right)$, NOR $\left(71.1^{\circ} \mathrm{N}\right)$ and $\mathrm{KEV}\left(69.8^{\circ} \mathrm{N}\right)$ which show the most prominent Pc5 activity in Figs. 2 and 3 are marked in Figs. 4 and 5 with dashed thin black horizontal lines. KKetal2013 present some results from a spectral analysis for the 20 min periods where pulsations were most prominent during the two events. This analysis reveals that the most dominant frequencies in the Pc5 pulsations shifted from a lower frequency to a higher frequency in both cases. On $18 \mathrm{Jan}-$ uary the dominant frequency was $3.1 \mathrm{mHz}$ during $04: 10$ 04:50 UT and 5.2 $\mathrm{mHz}$ during 04:50-05:10 UT. On 19 February the dominant frequencies were $4.0 \mathrm{mHz}$ and $4.7 \mathrm{mHz}$ for periods 05:05-05:25 and 05:25-05:45 UT. On 18 January the shift in the frequency was associated also with a change of the resonant latitude from $71^{\circ}$ MLAT (BJN station) to $68^{\circ}$ MLAT (NOR station). All these changes can be seen also in Figs. 4 and 5, and in the following discussion on 2-D equivalent current patterns we have divided the events into some 


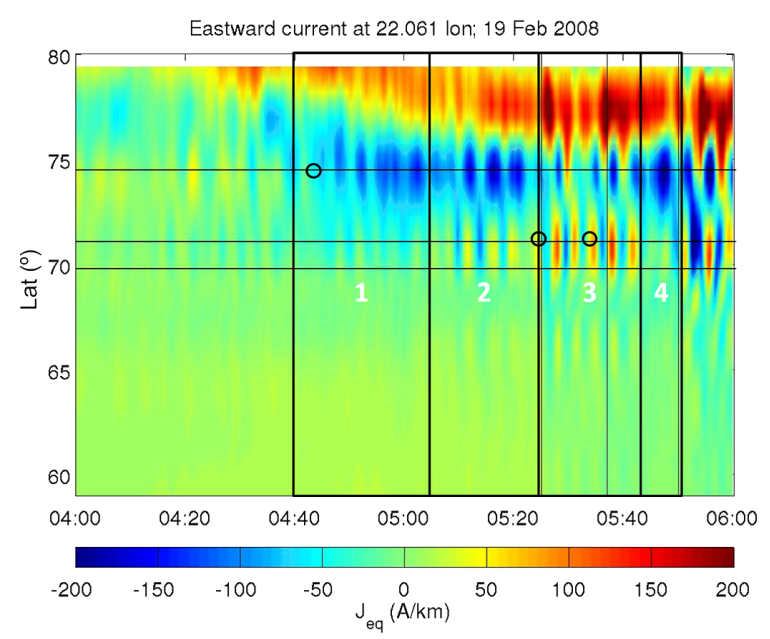

Figure 5. Eastward equivalent currents along the geographic meridian $22.1^{\circ}$ E during 04:00-06:00 UT on 19 February 2008. (The vertical axis is in geographical latitudes.) The four periods with different characteristics in 2-D equivalent current patterns are marked with black boundaries and white numbering. The geographic latitudes of stations BJN, NOR, and KEV are marked with the black horizontal lines. The auroral breakups at midnight sector took place at the end of Period 2 and at 05:37 (thin black vertical line). The time instants of the 2-D equivalent current maps of Fig. 8 are shown with the black circles.

sub-periods whose timing matches with the results of spectral analysis by KKetal2013.

Figures 6 and 8 show some examples of 2-D equivalent current patterns which are typical for the sub-periods described below in more detail (and marked numbers in Figs. 4 and 5).

\subsection{2-D equivalent current patterns on 18 January 2008}

\subsubsection{4:00-04:10 UT (Period 1 in Fig. 4, "background conditions")}

A clockwise $(\mathrm{CW})$ vortex on the southern or south-western side of Svalbard is the most typical equivalent current pattern observed during our pulsation events. As explained above, such a pattern can be associated with downward field-aligned currents. As Svalbard magnetometers were collecting data from the high-latitude morning sector, a likely explanation for the tendency of downward FACs to dominate in our current patterns is the presence of large-scale Region 1 (R1) currents in the analysis area. R1 current in the morning sector flows downward. During Period 1 the vortex was rather stable in its intensity and location. Equivalent currents in the Fennoscandia mainland (at stations NOR and KEV) remained weak throughout this period.

\subsubsection{4:11-04:50 UT (Period 2 in Fig. 4, "FLR resonance at $71^{\circ}$ MLAT")}

The intensity and size of the CW vortex on the south-west side of Svalbard (cf. Fig. 6a) varied quasi-periodically during 04:11-04:50 UT. Occasionally, when the CW vortex was weak and small, patterns of three simultaneous vortices with opposing rotation directions could be observed. Figure $6 \mathrm{~b}$ shows an example of such pattern: a CW vortex south-west of Svalbard, counterclockwise (CCW) vortex between Svalbard and BJN (station marked with a gray square in the Arctic Sea) and a CW vortex between BJN and mainland. Unfortunately, the vortex structures were not in the region of densest magnetometer network, but we can see that the triple vortex structure is confirmed by oppositely directed equivalent currents in the close vicinity of some critically located magnetometer stations. Equivalent currents in the Fennoscandian mainland (at stations NOR and KEV) were somewhat stronger than during Period 1 and their directions had some variations, like Fig. 4 demonstrates.

\subsubsection{4:51-05:09 UT (Period 3 in Fig. 4, "FLR resonance at $68^{\circ}$ MLAT")}

During 04:51-05:09 UT the CW vortex in the Svalbard was accompanied by another vortex located between BJN and mainland. The $\mathrm{CCW}$ and $\mathrm{CW}$ directions varied in the latter vortex, with $\mathrm{CCW}$ vortex being typically more pronounced (cf. Fig. 6c) than the $\mathrm{CW}$ one (Fig. 6d). The equivalent current intensities at the latitudes of KEV and NOR were stronger than earlier during this period. Some weaker vorticity were recorded also at lower latitudes (geographical latitudes $67-70^{\circ} \mathrm{N}$, cf. Fig. 6c).

\subsubsection{5:10-05:14 UT (Period 4 in Fig. 4, "Mode change")}

Some dramatic changes in the equivalent current patterns took place during 05:10-05:14 UT. A strong CW vortex appeared in the region between BJN and mainland. Inspection of equivalent patterns in higher time resolution $(10 \mathrm{~s})$ reveals that this vortex expanded westward (i.e., from dawn towards midnight, cf. Fig. 6e and f). As longitudinal coverage of magnetometer stations is very limited in the $\mathrm{CW}$ vortex region, it is difficult to say whether the vortex propagated to the analysis region from the prenoon sector or whether the structure just built up on the spot. However, it seems very unlikely that the structure came to the region from its western side, because magnetometers in Norwegian and Swedish mainland did not observe it before Finnish magnetometers.

In the context of this new vortex the auroras brightened and several arcs with vortex structures, smaller than the equivalent current vortex, were recorded by the all-sky camera at NAL (cf. Fig. 7). These auroras are similar to those presented in the FLR study by Samson et al. (1996). Si- 

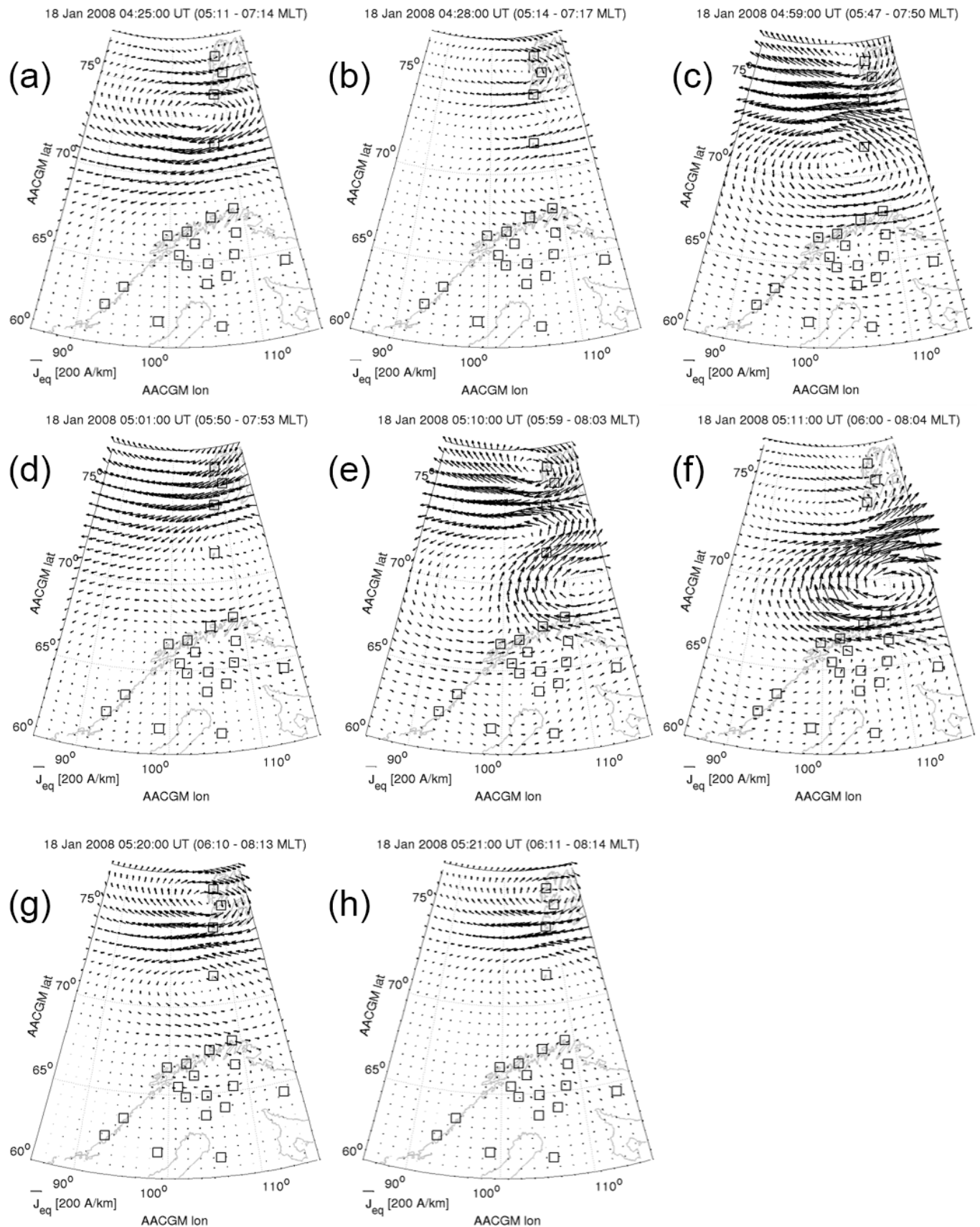

Figure 6. Examples of 2-D equivalent current plots derived from IMAGE magnetometer data recorded on 18 January 2008. Maps are presented in altitude-adjusted corrected geomagnetic coordinates (AACGMs, Baker and Wing, 1989).

multaneously with the new current vortex there was a small CCW (upward FAC) in the Svalbard region. The CCW vortex disappeared after a couple of minutes and at 05:14 UT the more typical $\mathrm{CW}$ vortex dominated the picture again, although now on the southern side of Svalbard.

\subsubsection{5:15-05:30 UT (Period 5 in Fig. 4, "Pc5 weakening")}

The $\mathrm{CW}$ vortex from the end of Period 4 moved to its more typical place, south-west of Svalbard, at the beginning of Period 5 (05:15-05:30 UT). A double vortex pattern resembling the one in Fig. 6c (but with weaker currents, cf. Fig. 6g) appeared periodically. During periods when this pattern was 

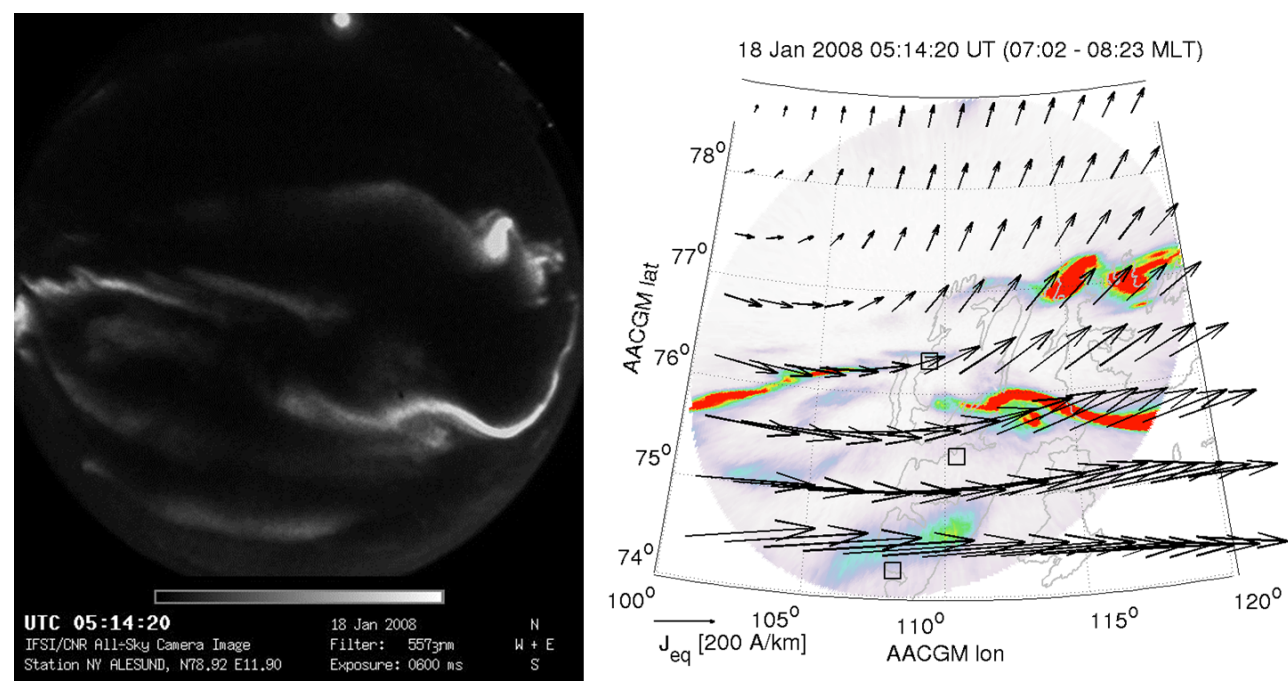

Figure 7. (left) All-sky camera image from the NAL station $(557.7 \mathrm{~nm})$ on 18 January 2008, at 05:14:20 UT and (right) its projection on a map (altitude $110 \mathrm{~km}$ assumed) together with some equivalent current vectors.

missing, the equivalent currents were small outside Svalbard (Fig. 6h) and had variable directions without clear systematic behavior. The absence of red and blue blobs at the BJN latitude in Fig. 4 during this period is explained partly by the occurrence of north-south-directed currents above this station.

\subsection{2-D equivalent current patterns on 19 February 2008}

The equivalent current patterns of 19 February repeat almost the same behavior as on 18 January. CW vortex south or south-west of Svalbard is again the most typical structure in these patterns. On 19 February the short Pc5 fading period was not preceded by such dramatic variations in current patterns as in the case of 18 January. As reference material for the 18 January event we describe briefly also the subperiods of 19 February below.

\subsubsection{4:40-05:05 UT (Period 1 in Fig. 5, "Polar cap expansion")}

Similarly as in the beginning of the 18 January event, a relatively stable $\mathrm{CW}$ vortex resided in the region south or southwest of Svalbard (example in Fig. 8a).

\subsubsection{5:06-05:25 UT (Period 2 in Fig. 5, "Pc5 of 4.0 mHz")}

The CW vortex in Svalbard region conducted quasiperiodic intensity variations. Occasionally a CCW vortex appeared in the region between $\mathrm{BJN}$ and the mainland (example in Fig. 8b).

\subsubsection{5:26-05:43 UT (Period 3 in Fig. 5, "Pc5 of $4.7 \mathrm{mHz}$ ")}

It is the same as above with the addition of occasional triplevortex patterns with a small CCW vortex above Svalbard (example in Fig. 8c).

\subsubsection{5:43-05:50 UT (Period 4 in Fig. 5, "Pc5 weakening")}

There is a stable CW vortex between Svalbard and BJN (resembles Period 1 of this event).

\section{Geotail and THEMIS P2 and P5 measurements}

\subsection{Solar wind dynamics pressure and IMF $B_{z}$}

Geotail satellite measured the solar wind conditions near the magnetopause at $X_{\mathrm{GSM}} \sim 26 R_{\mathrm{e}}$ and $X_{\mathrm{GSM}} \sim 18 R_{\mathrm{e}}$ for 18 January and 19 February, respectively. Magnetic field and plasma measurements were conducted with the magnetic field (MGF) and low-energy particle (LEP) experiments, respectively (Mukai et al., 1994; Kokubun et al., 1994). From LEP both the LEP-solar wind (LEP-SW) and LEP-EA (lowenergy particle experiment, energy-per-charge analyzer) instruments were used, from which the latter is designed to measure low densities prevailing typically in the magnetosphere.

As the solar wind speed was high during both events, the delays for the solar wind changes to propagate from Geotail to the magnetopause (at $\sim 10 R_{\mathrm{e}}$ ) were only some $1-3 \mathrm{~min}$. Estimating the delay time from the magnetopause to ionosphere is not straightforward as it depends on the Alfvén velocity in the magnetosphere, which varies considerably along 

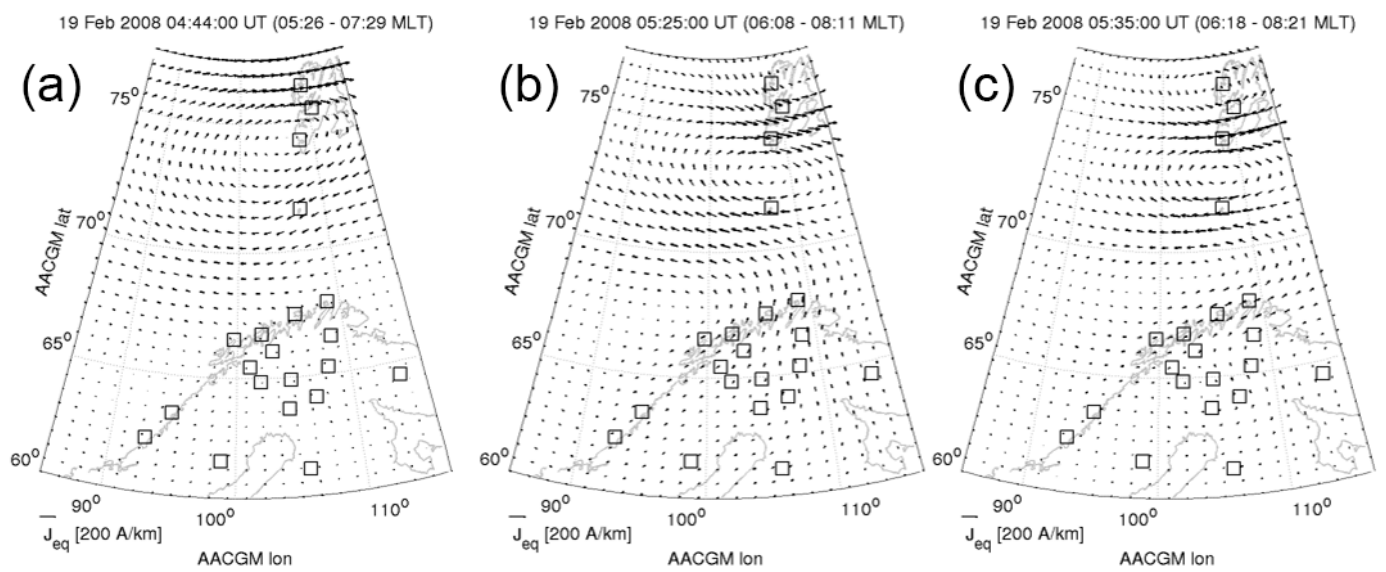

Figure 8. Examples of 2-D equivalent current plots derived from IMAGE magnetometer data recorded on 19 February 2008.

the signal propagation path. The literature presents different estimates for this delay. In the approach used in derivation of the polar cap index a delay of $20 \mathrm{~min}$ is assumed (Stauning, 2007) while Clauer and Banks (1986) concluded, from the basis of simulation results, that the propagation delay is $\sim 14 \mathrm{~min}$. We use the latter estimate in our analysis for shifting the times of the periods presented in Figs. 4 and 5, when comparing them with changes in solar wind dynamic pressure and IMF $B_{z}$.

As Figs. 9 and 10 show (in GSM coordinates), dynamic pressure of solar wind varied mainly around $1 \mathrm{nPa}$ during both events, with one exception around 04:48-04:54 UT on 18 January, when the pressure dropped down to $0.2 \mathrm{nT}$. This pressure drop is associated with a transient drop in the ion density which was measured by the LEP-EA instrument and with a velocity drop from 700 to $600 \mathrm{~km} \mathrm{~s}^{-1}$. Finding any systematic connection between IMF $B_{z}$ variations and changes in the pulsation characteristics is difficult, particularly in the case of 18 January, but we note that on 19 February the strongest pulsations were observed during Period 3 (and after Period 4), when IMF $B_{z}$ was mainly positive. So, at least in this particular case, the IMF $B_{z}$ direction seems to control the intensity of pulsations, which persisted throughout the analysis period, but were weaker when IMF $B_{z}$ was negative. On 19 February the solar wind density was stable $\left(1.4-1.5 \mathrm{~cm}^{-3}\right)$ throughout the period of our interest.

\subsection{Gradient drift of electrons from the substorm injection region}

On 18 January, the locations of the THEMIS P5 and P2 probes were in GSM coordinates $\left(-8.9,-1.5,-3.4 R_{\mathrm{e}}\right.$ and $\left.-8.4,-7.3,-3.7 R_{\mathrm{e}}\right)$. Like Fig. 1 shows, the footpoint of $\mathrm{P} 5$ was in the vicinity of the substorm breakup region while that of P2 was roughly midway between the P5 footpoint and MIRACLE instruments. Figure 11 shows the electron number flux measurements by P2 and P5 for energy channels 90$279 \mathrm{keV}$. The delay of eastward drifting electrons in the chan-
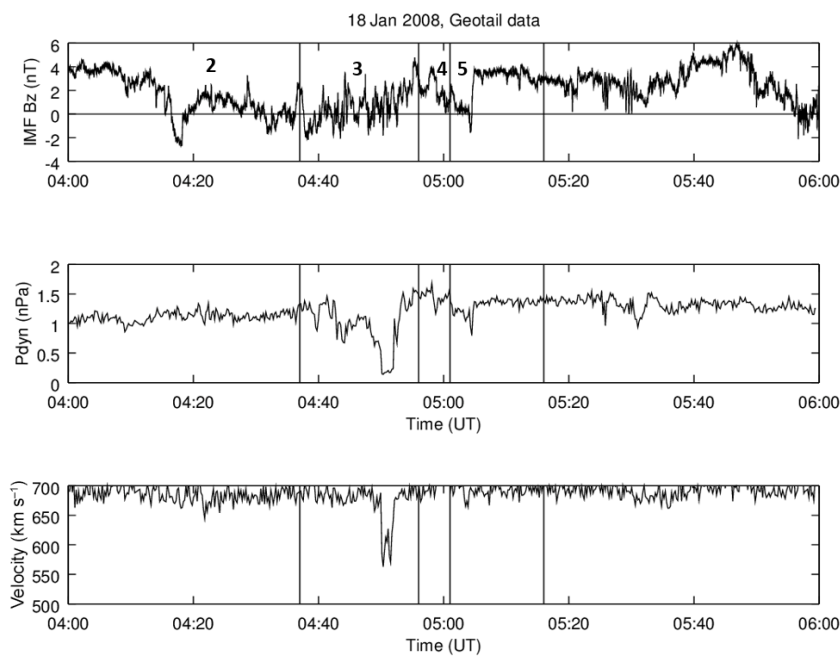

Figure 9. Geotail measurements of IMF Bz (top, GSM coordinates), dynamic pressure (middle), and velocity (bottom). The periods 2-5 from Fig. 4 are marked here with the black vertical lines and numbers with the assumption that the propagation time from the magnetopause to ionosphere is $14 \mathrm{~min}$.

nels $90-134 \mathrm{keV}$ from P5 to P2 is roughly $\sim 2 \min$ (when the times of flux maxima are compared), which gives an estimate of $26^{\circ} \mathrm{min}^{-1}$ (in magnetic longitude, cf. Fig. 1) for the azimuthal drift speed for these electrons. With this drift speed the electrons have reached the longitudes of MIRACLE network around 05:15+ 5 min, i.e., $\sim$ 05:20 UT. The electron flux values in the energy channel $279 \mathrm{keV}$ are an order of magnitude smaller than those in channels $90-134 \mathrm{keV}$, but clearly enhanced above their background level. For these electrons the delay from P5 to P2 is insignificant at the time resolution relevant for Pc5 studies. Therefore, this population most likely reached the morning sector around the time when Pc5 weakening started. This example demonstrates that energetic electrons drifting from the substorm injection region 

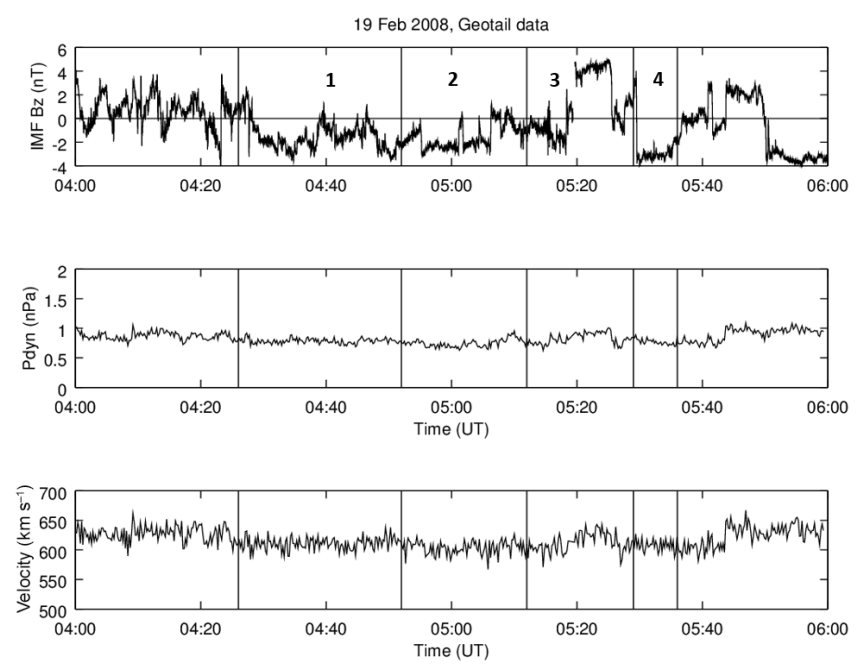

Figure 10. Geotail measurements of IMF Bz (top, GSM coordinates), dynamic pressure (middle), and density (bottom). The periods 1-4 from Fig. 5 are marked here with the black vertical lines and numbers with the assumption that the propagation time from the magnetopause to ionosphere is $14 \mathrm{~min}$.

can reach the morning sector in a few minutes, and thus they have potential to affect the Pc5 amplitudes there.

\section{Summary and concluding remarks}

We have studied the spatial and temporal distribution of equivalent currents in the morning sector of the auroral oval during two sequences of Pc5 pulsations (on 18 January and 19 February 2008) with FLR characteristics. Both events took place during periods of high solar wind speed $\left(>600 \mathrm{~km} \mathrm{~s}^{-1}\right.$ ), and thus they very much resemble the case of wave over-reflection at magnetopause as described in the model by Mann et al. (1999). In this scenario magnetosheath flows can extract energy from the background solar wind flow and feed it to the magnetospheric waveguide modes. A disturbance in the over-reflection mechanism would be a natural explanation for the temporal weakenings in Pc5 intensity which we describe in this study. As the solar wind speed remains high throughout our events, the cause must be somewhere else in the coupling process. With ionospheric equivalent currents we can probe magnetospheric processes in a wide latitude range if they are linked with the ionosphere, for example, with FACs, like they typically are in the case of FLRs. Equivalent currents have been derived from groundbased magnetometer network data with the method of SECSs (Amm 1997; Amm and Viljanen, 1999). We want to demonstrate that some information about the dynamics of FLRrelated FAC systems in a wider context can be deduced with the SECS method, although the impact of small-scale structures in ionospheric currents $(L \leq 50 \mathrm{~km})$ becomes smoothed out from ground-based magnetic observations (Untiedt and

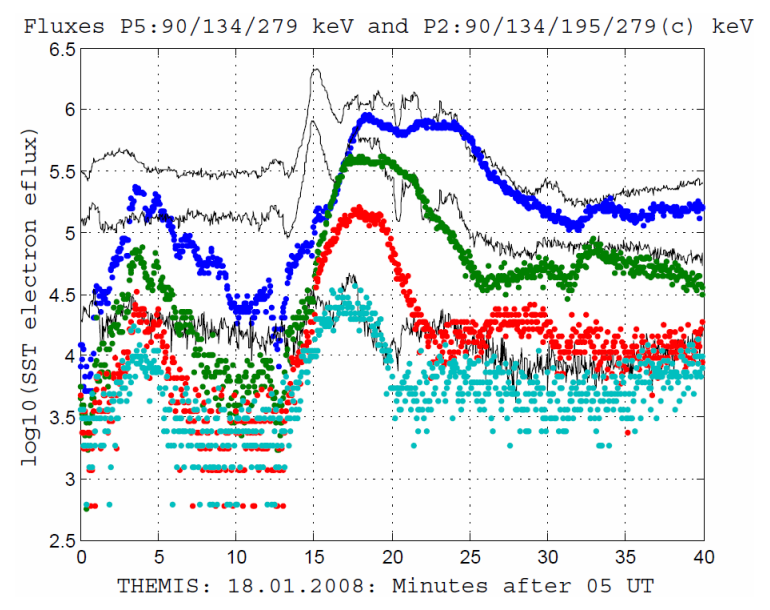

Figure 11. THEMIS P5 and P2 measurements of electron number flux. P5 measurements for channels 90, 134, and $279 \mathrm{keV}$ are shown with thin black lines (MLT 23). P2 measurements for channels $90,134,195$, and $279 \mathrm{keV}$ are shown with blue, green, red, and cyan dots (MLT 02). The weakening of Pc5 pulsations (05:0007:00 MLT) took place during 05:15-05:30 UT.

Baumjohann, 1993). Optical and radar measurements, when available, can describe FLR signatures with a better resolution (Milan et al., 2001).

In our events the most typical equivalent current pattern associated with Pc5 activity is a clockwise vortex, i.e., signature of a downward FAC with pulsating intensity and varying size and location. This vortex was at high latitudes (MLATs $74-75^{\circ}$ ), which gives us reason to believe that the vortex is a combined signature of morning-sector large-scale R1 and FLR FACs. Meridional sequences of two or three vortices with opposing rotation directions (upward and downward currents) which are more consistent with previously reported FLR FAC systems (Scoffield, et al., 2005; McPherron, 2005) appear in our equivalent current patterns too, but also in these structures the most poleward FAC was typically downward, i.e., parallel to morning-sector R1.

The changes in resonant frequency and latitude which the traditional FLR analysis (see, e.g., Ziesolleck et al., 1998; Samson et al., 1992; Mann and Wright, 1999) reveals for the two pulsation periods are visible as abrupt changes in the equivalent current patterns, as well. The shifts to higher resonant frequencies are associated with intensifications of equivalent current vortices at lower latitudes which represent footpoints of shorter field lines. The latitudinal distribution of magnetometer stations used in this study sets a limit for the accuracy with which we can determine the location of the resonant latitude. Within this limit, both the traditional analysis and SECS analysis locate the resonant latitudes to the same regions (poleward of BJN and NOR stations, white dashed lines in subperiods 2, 3 of Fig. 4). The longitudinal distribution of MIRACLE magnetometer stations allows estimates of azimuthal wave number $(m)$ and 
phase speed at two latitudes, MLAT 66.4 and $64.5^{\circ}$, with maximal longitudinal separation of 9 and $12^{\circ}$, respectively. Analysis of the $Y$-component magnetograms yields $m \sim 7-8$ and westward azimuthal phase speed of $11 \mathrm{~km} \mathrm{~s}^{-1}$ (18 January, $\sim 05: 10)$. The combination of solar wind speed and phase speed in our event is similar to those observed by Mathie and Mann (2000) in the case where the azimuthal phase speed can be associated with the propagation speed of shear flow instability at the magnetopause (cf. Fig. 11 of Mathie and Mann, 2000).

The two pulsation periods which we have analyzed occurred during substorm periods, and the Pc5 intensity in the morning sector showed some abrupt changes in the association of substorm breakups or auroral intensifications in the midnight sector. In particular, temporal weakenings of Pc5 activity were observed some minutes after breakup times in both cases. The potential connection between morning-sector Pc5 weakening and midnight substorm activity is discussed by KKetal2013. In their discussion on the potential reasons for this decrease three mechanisms are presented: (i) the substorm breakups change the global topology of magnetospheric magnetic field so that the conditions for field-line resonances disappear although KHI at the magnetopause would still maintain wave activity, or (ii) enhanced auroral precipitation in the morning sector changes the conductance (and electric field) at the footpoints of the resonating field lines so that they cannot maintain the standing wave oscillation anymore, or (iii) energetic particle injection to inner magnetosphere (Baker et al., 2002; Li et al., 2003) or energetic electrons leaking from outer parts of radiation belt (Borovsky and Denton, 2011) cause changes in magnetospheric resonance conditions.

The analysis of the equivalent current patterns and THEMIS satellite data conducted in this study reveals that the weakening of Pc5 pulsation on 18 January may be explained with two different scenarios, one triggered by changes in solar wind and the other related to substorm activity:

- According to Geotail data the solar wind dynamic pressure had a sudden drop at 04:48-04:52 UT, which most likely caused the sudden change in our equivalent current vector patterns from FLR-related smaller vortices to one single large $\mathrm{CW}$ vortex propagating antisunward. We associate the large vortex with a new downward directed FAC which built up as a response to the sudden pressure drop. These processes could disturb the energy transfer between the KHI at the magnetopause and magnetospheric FLRs and consequently also cause the weakening of Pc5 activity during a period of $\sim 15 \mathrm{~min}$ after the appearance of the new FAC.

- Our re-analysis of THEMIS satellite data reveals that we cannot totally exclude the midnight substorm activity from the factors affecting morning-sector Pc5 activity. The substorm breakup observed at the midnight sector was associated with a population of energetic electrons $(\sim 200 \mathrm{keV})$ which most likely reached the morning-sector flux tubes by the time of Pc5 weakening. Therefore it is possible that this new particle population modulated either the content of flux tubes or the conductance at their footpoints so that the resonant activity ceased.

The statistics by Baker et al. (2003) show increased Pc5 occurrence for IMF $B_{z}>0$, which is consistent with our data collected during the second pulsation period on 19 February 2008. In that case a likely explanation for the Pc5 weakening comes from IMF $B_{z}$ turning to negative values. We cannot use P2 to analyze eastward-drifting electrons similarly as done above for the case of 18 January, because on 19 February the THEMIS probes were in the evening and midnight sector of the oval. Surprisingly, also in this case a short-lived enhancement of energetic electrons was observed in the evening sector by P2 (simultaneously with an energy dispersed enhancement in ion fluxes) at 05:25 UT, i.e., around the time of auroral breakup in the midnight sector and of a change in the most dominant FLR frequency in the morning sector. The observation of simultaneous enhancements in energetic electron fluxes both in the midnight and evening sector resembles the substorm case reported by Blake et al. (2005), where bursts of energetic electrons were observed at several local times and radial distances simultaneously $\sim 10$ min after the substorm onset. The authors interpret their observations as a reconfiguration of the magnetosphere driven by similar changes in IMF $B_{z}$ direction as observed in our case. It is clear, however, that IMF $B_{z}$ changes alone cannot generate these reconfigurations as globally distributed bursts of energetic electrons are rarely observed in the magnetotail.

Like explained above, on 18 January the Pc5 weakening was preceded by a 4 min period when a westward-expanding, strong $\mathrm{CW}$ vortex replaced the Pc5-related equivalent current pattern with two smaller vortices in the morning sector. The new vortex was at closed field lines, and it was occasionally accompanied by a small CCW vortex (upward current) which appeared at higher latitudes in the region where typically the downward R1 currents reside. After the 4 min period, similar equivalent current patterns were recorded as before the $\mathrm{CW}$ vortex intrusion, but the equivalent current intensities were smaller than for the periods of clear FLR activity. We also want to point out that a part of the Pc5 weakening which is visible in the filtered magnetometer data (Fig. 2) may be due to non-optimal distribution of our magnetometer stations. As the equivalent current patterns of Pc5 are localized in longitude, a shift of the current system may appear as weakening in the pulsation amplitude when measured with a single station.

With the available observations is it is difficult to determine which mechanism in the magnetosphere caused the new CW vortex on 18 January. Traveling convection vor- 
tices (TCVs) and flux transfer events (FTEs) are examples of such localized dayside structures which could cause similar ionospheric signatures as were observed in our case. As FTEs appear at open field lines, a TCV would be a more plausible candidate to explain the $\mathrm{CW}$ vortex of our case. According to Moretto and Yahnin (1998) the FACs of TCVs build up deep inside of magnetosphere. It is possible that our regional magnetometer network cannot see the entire twinvortex pattern which in the case of TCVs can extend over $80^{\circ}$ in longitude (Amm et al., 2002), but on the other hand we did not see the twin-vortex pattern drifting across our network similarly as in some earlier TCV studies. Also, the values of solar wind dynamic pressure in our case - in the range from $\sim 1$ to $0.2 \mathrm{nPa}$ - are different from those of typical TCV conditions (e.g., Friis-Christensen et al., 1988). The minimum of $0.2 \mathrm{nPa}$ is associated with a velocity drop from 700 to $600 \mathrm{~km} \mathrm{~s}^{-1}$ and with drop of solar wind density from values above $1 \mathrm{~cm}^{-3}$ to values around $0.3 \mathrm{~cm}^{-3}$. The low density values were measured by the LEP-EA instrument of Geotail, which is designed to measure low densities prevailing typically in the magnetosphere (Mukai et al., 1994). We believe that the cavity of low density values in solar wind, which caused the switch from the standard solar wind instrument (LEP-SW) to LEP-EA, was also able to generate the antisunward-propagating FAC system which resembled a $\mathrm{TCV}$, but was most likely smaller in spatial dimensions and had a shorter lifetime.

Our case studies demonstrate that both solar wind changes and substorm activity can cause variations in the intensity of morning-sector Pc5 pulsations. Regional equivalent current patterns appeared to be a useful way to study the impact of solar wind changes, while particle measurements in the magnetotail are needed to observe the linkage between substorm onsets and Pc5 activity. An interesting topic for future statistical studies could be to check whether there are systematic differences in the Pc5-related equivalent current patterns as response to different driver mechanisms (solar wind ULF waves vs. KHI at magnetopause). The ECLAT archive of 2-D equivalent current data available in the Cluster Active Archive and the OMNI solar wind database will be valuable assets to facilitate such work.

Acknowledgements. The work of N. G. Kleimenova was supported by the Program RAS No. 7. The work of S. V. Dubyagin was supported by the Finnish Academy of Sciences. L. Juusola is acknowledged for fruitful discussions and coordinate transformations.

The work of paper Kauristie et al. (2013) (KKetal2013) and this paper is based on observations from several ground-based instrument networks and satellites.

We acknowledge NASA contract NAS5-02099 and V. Angelopoulos for use of data from the THEMIS Mission - specifically, J. W. Bonnell and F. S. Mozer for use of EFI data; C. W. Carlson and J. P. McFadden for use of ESA data; D. Larson and R. P. Lin for use of SST data; K. H. Glassmeier, U. Auster and W. Baumjohann for the use of FGM data provided under the lead of the Technical
University of Braunschweig and with financial support through the German Ministry for Economy and Technology and the German Aerospace Center (DLR) under contract 50 OC 0302; S. Mende and E. Donovan for use of the ASI data, the CSA for logistical support in fielding and data retrieval from the GBO stations, and NSF for support of GIMNAST through grant AGS-1004736; I. R. Mann, D. K. Milling and the rest of the CARISMA team for use of GMAG data. CARISMA is operated by the University of Alberta, funded by the Canadian Space Agency; Troms $\emptyset$ Geophysical Observatory, University of Troms $\varnothing$, Norway, and DTU, Denmark, for use of the Greenland and Norway magnetometer data. The SNK station is operated by Natural Resources Canada.

Geotail magnetic field and plasma data were provided by T. Nagai and Y. Saito through DARTS at Institute of Space and Astronautical Science, JAXA in Japan.

EISCAT is an international association supported by research organizations in China (CRIRP), Finland (SA), Japan (NIPR and STEL), Norway (NFR), Sweden (VR), and the United Kingdom (NERC).

The MIRACLE network is operated as an international collaboration under the leadership of the Finnish Meteorological Institute. The IMAGE magnetometer data are collected as a joint European collaboration. CNR/Italy maintains the ITACA ASC in NyÅlesund.

The topical editor, G. Balasis, thanks M. Connors and one anonymous referee for help in evaluating this paper.

\section{References}

Akasofu, S.-I.: The development of the auroral substorm, Planet. Space Sci., 12, 273-282, 1964.

Amm, O.: Ionospheric elementary current systems in spherical coordinates and their application, J. Geomagn. Geoelectctr., 49, 947-955, 1997.

Amm, O. and Viljanen, A: Ionospheric disturbance magnetic field continuation from the ground to ionosphere using spherical elementary current systems, Earth Planets Space, 51, 431-440, 1999.

Amm, O., Engebretson, M. J., Hughes, T., Newitt, L., Viljanen, A., and Watermann, J.: A traveling convectin vortex event study: Instantaneous ionospheric equivalent currents, estimation of field aligned currents, and the role of induced currents, J. Geophys. Res., 107, 1334, doi:10.1029/2002JA009472, 2002.

Angelopoulos, V.: The THEMIS mission, in: The THEMIS mission, edited by: Burch, J. L. and Angelopoulos, V., Springer New York, ISBN: 978-0-387-89820-9, doi:10.1007/978-0-38789820-9_2, 2009.

Baker, K. B. and Wing, S.: A new magnetic coordinate system for conjugate studies at high latitudes, J. Geophys. Res., 94, 91399143, 1989.

Baker, D. N., Peterson, W. K., Eriksson, S., Li, X., Blake, J. B., Burch, J. L., Daly, P. W., Dunlop, M. W., Korth, A., Donovan, E., Friedel, R., Fritz, T. A., Frey, H. U., Mende, S. B., Roeder, J., and Singer, H. J.: Timing of magnetic reconnection initiation during a global magnetospheric substorms onset, Geophys. Res. Lett., 29, 2190-2193, doi:10.1029/2002GL015539, 2002. 
Baker, G. J., Donovan, E. F., and Jackel, B. J.: A comprehensive survey of auroral latitude Pc5 pulsation characteristics, J. Geophys. Res., 108, 1384, doi:10.1029/2002JA009801, 2003.

Blake, J. B., Mueller-Mellin, R., Davies, J. A., Li, X., and Baker, D. N.: Global observations of energetic electrons around the time of a substorm on 27 August 2001, J. Geophys. Res., 110, A06214, doi:10.1029/2004JA010971, 2005.

Borälv, E., Eglitis, P., Opgenoorth, H. J., Donovan, E., Reeves, G., and Stauning, P.: The dawn and dusk electrojet response to substorm onset, Ann. Geophys., 18, 1097-1107, doi:10.1007/s00585-000-1097-4, 2000.

Borovsky, J. E. and Denton, M. H.: Evolution of the magnetotail energetic electron population during high-speed-stream-driven storms: Evidence for the leakage of the outer electron radiation belt into the Earth's magnetotail, J. Geophys. Res., 116, A12228, doi:10.1029/2011JA016713, 2011.

Clauer, C. R. and Banks, P. M.: Relationship of the interplanetary electric field to the high-latitude ionospheric electric fields and currents: Observations and model simulation, J. Geophys. Res., 91, 6959-6971, 1986.

Connors, M., Rostoker, G., Sofko, G., McPherron, R. L., and Henderson, M. G.: Ps 6 disturbances: relation to substorms and the auroral oval, Ann. Geophys., 21, 493-508, doi:10.5194/angeo21-493-2003, 2003.

Fenrich, F. R. and Waters, C. L.: Phase coherence analysis of a field line resonance and solar wind oscillation, Geophys. Res. Lett., 35, L21002, doi:10.1029/2008GL035430, 2008.

Friis-Christensen, E., Kamide, Y., Richmond A. D., and Matsushita, S.: Interplanetary magnetic field control of highlatitude electric fields and currents determined from Greenland Magnetometer Data, J. Geophys. Res., 90, 1325-1338, doi:10.1029/JA090iA02p01325, 1985.

Friis-Christensen, E., McHenry, M. A., Clauer, C. R., and Vennerström, S.: Ionospheric traveling convectin vortices observed near the polar cleft: A triggered response to sudden changes in the solar wind, Geophys. Res. Lett., 15, 253-256, 1988.

Hagfors, T.: Incoherent scatter radar observations of the plasma line with a chirped pulse system, Radio Sci., 17, 727-734, 1982.

Hubert, B., Aikio, A. T., Amm, O., Pitkänen, T., Kauristie, K., Milan, S. E., Cowley, S. W. H., and Gérard, J.-C.: Comparison of the open-closed field line boundary location inferred using IMAGEFUV SI12 images and EISCAT radar observations, Ann. Geophys., 28, 883-892, doi:10.5194/angeo-28-883-2010, 2010.

Juusola, L., Amm, O., and Viljanen, A.: One-dimensional spherical elementary current systems and their use for determining ionospheric currents from satellite measurements, Earth Planets Space, 58, 667-678, 2006.

Juusola, L., Nakamura, R., Amm, O., and Kauristie, K.: Conjugate ionospheric equivalent currents during bursty bulk flows, J. Geophys. Res., 114, A04313, doi:10.1029/2008JA013908, 2009.

Juusola, L., Milan, S. E., Lester, M., Grocott, A., and Imber, S. M.: Interplanetary magnetic field control of the ionospheric fieldaligned current and convection distributions, J. Geophys. Res.Space, 119, 3130-3149, doi:10.1002/2013JA019455, 2014.

Kauristie, K., Uspensky, M. V., Kleimenova, N. G., Kozyreva, O., Dubyagin, S. V., and Vlasov, A. A.: Isolated Nighttime substorms and morning geomagnetic Pc5 pulsations from ground-based and satellite (THEMIS) observations, Geomagn. Aeronomy+, 53, 613-625, 2013.
Kepko, L., Spence, H. E., and Singer, H. J.: ULF waves in the solar wind as direct drivers of magnetospheric pulsations, Geophys. Res. Lett., 29, 1197, doi:10.1029/2001GL014405, 2002.

Kleimenova, N. G., Kozyreva, O. V., Manninen, J., and Ranta, A.: Unusual strong quasi-monochromatic ground Pc5 geomagnetic pulsations in the recovery phase of November 2003 superstorm, Ann. Geophys., 23, 2621-2634, doi:10.5194/angeo-232621-2005, 2005.

Kokubun, S., Yamamoto, T., Acuna, M. H., Hayashi, K., Shiokawa, K., and Kawano, H.: The GEOTAIL Magnetic field experiment, J. Geomag. Geoelectr., 46, 7-21, 1994.

Koskinen, H. E. J., Pulkkinen, T. I., and Pellinen, R. J.: Mapping of the auroral horn into the magnetotail, Planet. Space Sci., 38, 1179-1186, 1990.

Li, X., Baker, D. N., Temerin, M., Reeves, R. D., and Belian R. D.: Simulation of dispersionless injections and drift echoes of energetic electrons associated with substorms, Geophys. Res., Lett., 25, 3763-3766, 1998.

Li., X., Sarris, T. E., Baker, D. N., Peterson, W. K., and Singer, H. J.: Simulation of energetic particle injections associated with a substorm on August 27, 2001, Geophys. Res. Lett., 30, 10041007, doi:10.1029/2002GL015967, 2003.

Mann, I. R. and Wright, A. N.: Diagnosing the Excitation Mechanisms of Pc5 Magnetospheric Flank Waveguide Modes and FLRs, Geophys. Res. Lett., 26, 2609-2612, 1999.

Mann, I. R., Wright, A. N., Mills, K. J., and Nakariakov, V. M.: Excitation of magnetospheric waveguide modes by magnetosheath flows, J. Geophys., Res., 104, 333-353, 1999.

Mann, I. R., Milling, D. K., Rae, I. J., Ozeke, L. G., Kale, A., Kale, Z. C., Murphy, K. R., Parent, A., Usanova, M., Pahud, D. M., Lee, E.-A., Amalraj, V., Wallis, D. D., Angelopoulos, V., Glassmeier, K.-H., Russell, C. T., Auster, H.-U., and Singer, H. J.: The Upgraded CARISMA Magnetometer Array in the THEMIS Era, Space Sci. Rev., 141, 413-451, doi:10.1007/s11214-008-9457-6, 2008.

Mathie, R. A. and Mann, I. R.: Observations of Pc5 field line resonance azimuthal phase speeds: A diagnostic of their excitation mechanism, J. Geophys. Res., 105, 10713-10728, 2000.

McPherron, R. L.: Magnetospheric substorms, Rev. Geophys., 17, 657, doi:10.1029/rg017i004p00657, 1979.

McPherron, R. L.: Magnetic pulsations: Their sources and relation to solar wind and geomagnetic activity, Surv. Geophys., 26, 545592, 2005.

Milan, S. E., Sato, N., Ejiri, M., and Moen J.: Auroral forms and field-aligned current structure associated with field line resonances, J. Geophys. Res., 106, 25825-25833, 2001.

Moretto, T. and Yahnin, A.: Mapping travelling convection vortex events with respect to energetic particle boundaries, Ann. Geophys., 16, 891-899, doi:10.1007/s00585-998-0891-2, 1998.

Mukai, T., Machida, S., Saito, Y., Hirahara, M., Terasawa, T., Kaya, N., Obara, T., Ejiri, M., and Nishida, A.: The Low Energy Particle (LEP) Experiment onboard the GEOTAIL Satellite, J. Geomag. Geoelectr., 46, 669-692, 1994.

Rostoker, G., Spadinger, I., and Samson, J. C.: Local time variations in the response of Pc5 pulsations in the morning sector to substorm expansive phase onsets near midnight, J. Geophys. Res., 89, 6749-6757, 1984.

Röttger, J.: Incoherent scatter observations of the auroral ionosphere with the EISCAT radar facility, in: Auroral Physics, edited by: 
Meng, C.-I., Rycroft, M. J., and Frank, L. A., 419-437, Cambrige University Press, 1991.

Samson, J. C. and Rostoker, G.: Response of dayside Pc5 pulsations to substorm activity in the nighttime magnetosphere, J. Geophys. Res., 86, 733-752, 1981.

Samson, J. C., Harrold, B. G., Ruohoniemi, J. M., Greenwald, R. A., and Walker, A. D. M.: Field line resonances associated with MHD waveguides in the magnetosphere, Geophys. Res. Lett., 19, 19441-19444, 1992.

Samson, J. C., Cogger, L. L, and Pao, Q.: Observations of field line resonances, auroral arcs, and auroral vortex structures, J. Geophys. Res., 101, 17373-17383, 1996.

Scoffield, H. C., Yeoman, T. K., Wright, D. M., Milan, S. E., Wright, A. N., and Strangeway, R. J.: An investigation of the field-aligned currents associated with a large-scale ULF wave using data from CUTLASS and FAST, Ann. Geophys., 23, 487498, doi:10.5194/angeo-23-487-2005, 2005.

Sergeev, V., Kubyshkina, M., Alexeev, I., Fazakerley, A., Owen, C., Baumjohann, W., Nakamura, R., Runov, A., Vörös, Z., Zhang, T. L., Angelopoulos, V., Sauvaud, J.-A., Daly, P., Cao, J. B., and Lucek, E.: Study of near-Earth reconnection events with Cluster and Double Star, J. Geophys. Res., 113, A07S36, doi:10.1029/2007JA012902, 2008.

Stauning, P.: A new index for the interplanetary merging electric field and geomagnetic activity: Application of the unified polar cap indices, Space Weather, 5, S09001, doi:10.1029/2007SW000311, 2007.

Stephenson, J. A. E. and Walker, A. D. M.: Coherence between radar observations of magnetospheric field line resonances and discrete oscillations in the solar wind, Ann. Geophys., 28, 4759, doi:10.5194/angeo-28-47-2010, 2010.
Syrjäsuo, M. T., Pulkkinen, T. I., Janhunen, P., Viljanen, A., Pellinen, R. J., Kauristie, K., Opgenoorth, H. J., Wallman, S., Eglitis, P., Karlsson, P., Amm, O., Nielsen, E., and Thomas, C.: Observations of substorm electrodynamics using the MIRACLE network, in: Substorms-4, edited by: Kokubun, S. and Kamide, Y., Terra Scientific Publishing Compary, Tokyo, 111-114, 1998.

Untiedt, J. and Baumjohann, W.: Studies of polar current systems using the IMS Scandinavian Magnetometer Array, Space Sci. Rev., 63, 245-390, 1993.

van de Kamp, M.: Harmonic quiet-day curves as magnetometer baselines for ionospheric current analyses, Geosci. Instrum Method. Data Syst., 2, 289-304, doi:10.5194/gi-2-289-2013, 2013.

Viljanen, A. and Häkkinen, L.: IMAGE magnetometer network, in: Satellite-Ground Based Coordination Sourcebook, edited by: Lockwood, M., Wild, M. N., and Opgenoorth, H. J., ESA publications SP-1198, 111-117, 1997.

Zelinsky, N. R., Kleimenova, N. G., and Malysheva, L. M.: Studies on the latitudinal distribution of ground-based geomagnetic pulsations and fluctuations in the interplanetary medium, Geomagn. Aeronomy+ (English ed.), 34, 449-455, 2014.

Ziesolleck, C. W. S. and McDiarmid, D. R.: Statistical survey of auroral latitude Pc5 spectral and polarization characteristics, J. Geophys. Res., 100, 19299-19312, 1995.

Ziesolleck, C. W. S., Fenrich, F. R., Samson J. C., and McDiarmid, D. R.: Pc5 field line resonance frequencies and structure observed by SuperDARN and CANOPUS, J. Geophys. Res., 103, 11771-11785, 1998. 